\title{
Effect of geometrical defects on the tensile properties of graphene
}

\author{
Yunpeng Ren and Guoxin $\mathrm{Cao}^{*}$ \\ HEDPS, Center for Applied Physics and Technology, Department of Mechanics and Engineering \\ Science, College of Engineering, Peking University, Beijing, 100871, China
}

\begin{abstract}
The tensile properties of graphene with the Stone-Wales $(\mathrm{S}-\mathrm{W})$ defect are investigated by first principle calculations. It is found that the defect will not affect the elastic modulus and Poisson's ratio of graphene but causes the pre-stress of graphene, which makes the graphene to be anisotropic: the deformation along one direction is much easier than that along another direction. The pre-stress field causes only about $10 \%$ decrease of the intrinsic stress of graphene but causes more than $50 \%$ decrease of the maximum failure strain, which is significantly different with the results calculated by the empirical potential. The main reason is that the pre-stress field created by the $\mathrm{S}-\mathrm{W}$ defect predicted by first principle calculations is different than that calculated from molecular mechanics simulations. In addition, it is also found that the theoretical solution (on the basis of the continuum disclination dipoles model) of the pre-stress field created by the S-W defect is also different with that determined from MM simulations, which is different from the results of the aligned 7-5 defects of graphene.
\end{abstract}

\section{Introduction}

Graphene has attracted extensive research investigations because of its particular electrical, thermal, chemical and mechanical properties.[1-7] The basic building blocks of graphene are the hexagonal rings (6-rings) of the $s p^{2}$-bonded carbon atoms located in the same plane. 
However, in real manufacturing, graphene always includes geometrical defects, such as vacancy[8-10], five- and seven-membered ring pairs (7-5 rings or 7-5 defect)[11, 12], Stone-Wales (S-W) defect [13-15], 7-5-5-7 defect [16-18], 5-8-5 defect [16, 19], and so on. In addition to the isolated defects, grain boundary (GB) is commonly found in large-area polycrystalline graphene [20,21], which is mainly formed by the connected 7-5 defects [22-24].

Pristine graphene is one of the strongest materials ever measured: having an ultrahigh elastic modulus of $\sim 1 \mathrm{TPa}$ and an intrinsic strength of $\sim 130 \mathrm{GPa}$.[6] The mechanical properties of polycrystalline graphene have been studied and the highly inconsistent results were reported. Lee et al [21] reported that the elastic stiffness of polycrystalline is identical to that of pristine graphene and the strength is slightly reduced due to the presence of GBs; whereas Huang et al[25] and Ruiz-Vargas et al[26] shown that GBs severely waken the mechanical strength of graphene. Rassol et al [27] recently found that GB strength is highly sensitive to its mismatch angle in polycrystalline graphene: GBs with a large angle have a comparable strength with single-crystal graphene, whereas GB strength rapidly decreases with the angle.

In addition to experimental works, theoretical and numerical works have also developed to show the GB effect on the strength of polycrystalline graphene[12, 13, 15, 28-39]. Based on molecular dynamics (MD) simulations of bicrystalline graphene, GB strength was firstly considered to be a function of mismatch angle[28, 29, 38]: GBs with large mismatch angle (with a higher density of 7-5 defects) are much stronger than those with low angle (with a 
lower density of 7-5 defects), whereas the abnormal cases for armchair-type graphene were then reported[28, 29]. It was shown that the distribution/arrangement of defects also affects GB strength due to the coupling effect of stress field created by 7-5 defects, which can be partially cancelled with each other for uniformly distributed defects but not for those non-uniformly distributed. Actually, for both uniformly and non-uniformly distributed defects, the GB strength is not directly related to the mismatch angle of GB but depends on the strength of the bond (belongs to one 7-5 defect) with the highest pre-tensile stress component along the loading direction (created by the other 7-5 defects)[28]. Moreover, Grantab et al [29] reported that the GB strength depends on the initial $\mathrm{C}-\mathrm{C}$ bond stretching ratio created by 7-5 defects: the higher the bond is per-stretched, the lower the strength.

Most of the aforementioned computational works are based on classic molecular dynamics (MD) simulations, in which the adaptive intermolecular reactive empirical bond-order (AIREBO) potential is used to simulate the mechanical failure of polycrystalline graphene under tension. However, the assumptions taken by the empirical potential might not accurately show the effect of defect on the failure behavior of graphene. For example, it is reported that the empirical potential gives the different values of the elastic modulus and Poisson's ratio of prefect graphene[13, 40] as well as its geometrical response under a large tensile load.[41, 42] Therefore, a comprehensive study based on first principle calculations is highly necessary to investigate the effect of geometrical defect on the tensile strength and failure strain of graphene, which can provide a useful guideline to understand the GB strength. To the best of our knowledge, this might be the first study to investigate the effect of 
geometrical defect on the tensile properties (including elastic modulus, intrinsic strength and failure strain) of graphene monolayer by using first principle calculations.

\section{Computational Methods}

In the present study, we investigated the effect of geometrical defects on the tensile strength and failure strain of graphene under the uniaxial/biaxial tension by density functional theory (DFT) calculations, which is performed using the Vienna Ab Initio Simulation Package (VASP) $[43,44]$. Since the number of atoms in the computational cell calculated by DFT is very low, the boundary effect will strongly affect the effectiveness of the simulation results. In order to use the periodical boundary condition to remove the boundary effect in DFT calculations, a pair of 7-5 defects with the opposite direction connected with each other (also called the S-W defect) are considered in the present work, which are introduced into the computational cell by a S-W bond rotation, as shown in Figure 1. Figure 1(b) and 1(c) show the defect structures obtained from the armchair (ac) and zigzag (zz) graphene, respectively, where $d_{1}$ and $d_{4}$ are along ac direction, $d_{2}$ and $d_{3}$ are along zz direction. The uniaxial/biaxial strain tension is applied by increasing the computational cell size along the loading direction. It has been verified that a larger computational cell size produces only a negligible change of the defect formation energy.

The exchange-correlation terms are calculated by the Perdew-Burke-Ernzerh (PBE) generalized gradient approximation (GGA). The computational cell size is $2.4692 \times 2.1384 \times$ $1.5 \mathrm{~nm}$, which includes 200 carbon atoms. A Monkhorst-Pack $k$-point mesh grid $2 \times 2 \times 1$ is selected, and the plane wave energy cut-off is set at $750 \mathrm{eV}$ [45]. The $\mathrm{C}-\mathrm{C}$ bond length is 
calculated as $0.1425 \mathrm{~nm}$ in the undeformed structure of perfect graphene, which is in good agreement with the reported results from both experimental and simulation works $(0.1421-0.1422 \mathrm{~nm})[46,47]$. The formation energy of a S-W defect (created by a S-W bond rotation) is $4.705 \mathrm{eV}$ (very close to the reported result[15]), which indicates that the size effect of computational cell can be neglected.

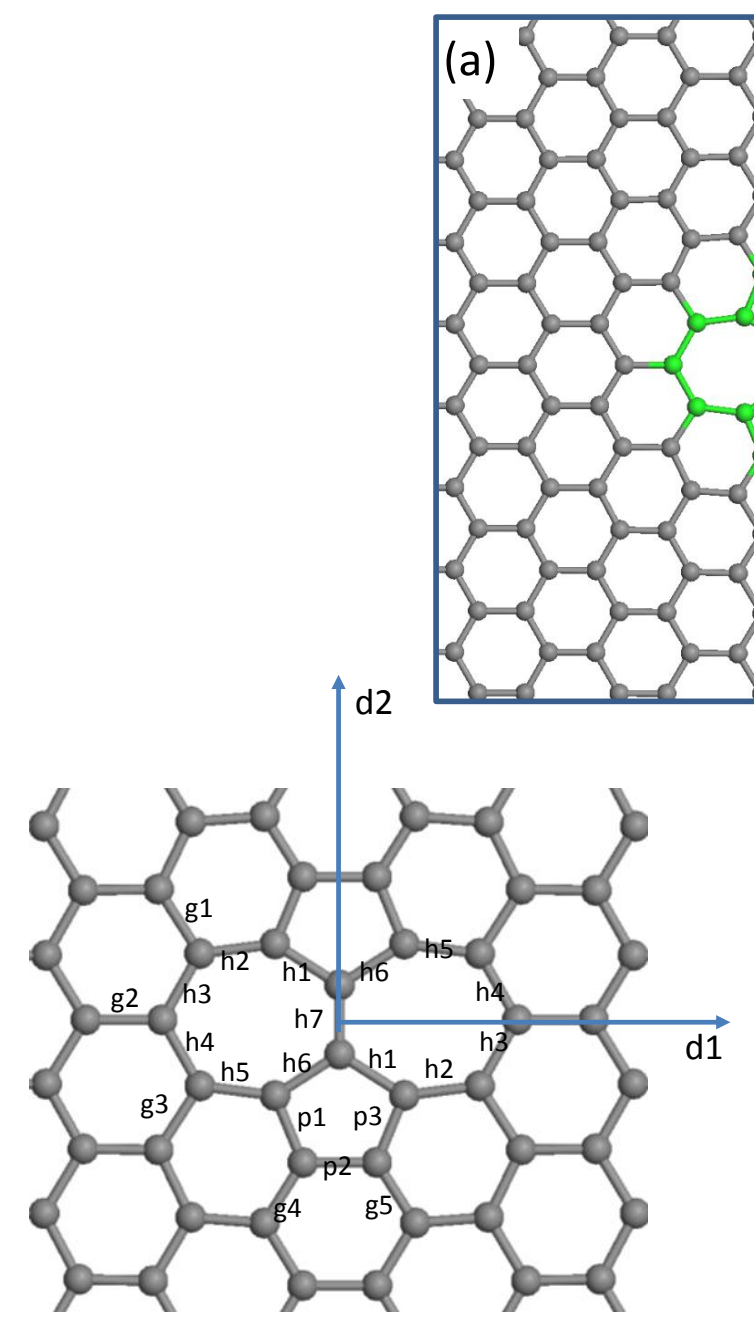

(b)

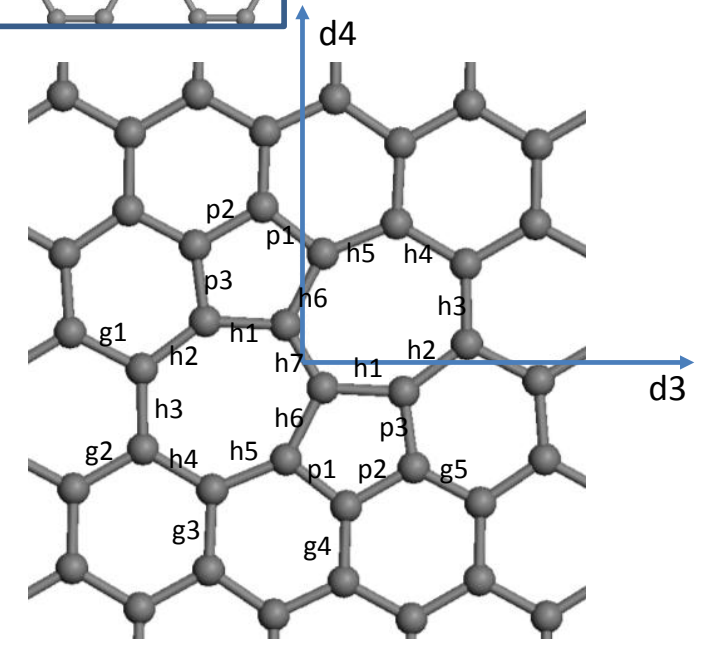

(c)

Figure 1. The computational model of graphene with a S-W defect in DFT calculations. (a) computational cell; (b) $x$-, $y$-axis along $d_{1}, d_{2}$ direction, respectively; (c) $x$-, $y$-axis along $d_{3}, d_{4}$ direction, respectively. The 7-ring includes h1-h7 C-C bonds; the 5-ring includes p1-p3, h1 and h6 bonds. Bonds g1-g5 are in the 6-rings close to the defect. 
In the present work, all MM simulations are carried out using the LAMMPS package[48] and a periodic boundary condition is applied in the lateral directions to remove the lateral boundary effect and simulate the intrinsic properties of graphene monolayer. In order to show the computational cell size effect, two different computational cells are selected: the smaller one has the same number of atoms as that used in DFT calculations; the larger one includes 1728 atoms and the size is about $6.53 \times 6.71 \times 2 \mathrm{~nm}$. The present $\mathrm{MM}$ simulation results are not sensitive to the computational cell. The REBO potential is used to optimize the initial/deformed structure of graphene, which has been widely used in studying carbon type materials.

\section{Results and Discussions}

\subsection{Effect of the defect on the elastic behavior of graphene}

Under uniaxial tension, the strain energy density $(u)$ of perfect graphene is invariant with an in-plane rotation of $\pi / 3$, and $u$ shows the same value along armchair (ac) and zigzag $(z z)$ directions with $\varepsilon<5 \%$ (i.e., perfect graphene is isotropic under a small deformation). After including a S-W defect, the structural symmetry is reduced (the invariant rotation angle of geometry changes to $\pi$ and the structure is strongly anisotropic (as shown in Figure 2). Figures 2 show the $u-\varepsilon$ relationship of graphene with a S-W defect (see Figure 1) under uniaxial strain tension, where $\varepsilon$ is the Lagrange tensile strain. To show the anisotropy, four different loading directions are selected: direction $1\left(d_{1}\right)$ is along two 7-rings, direction $2\left(d_{2}\right)$ is along two 5-rings, directions 3 and $4\left(d_{3}\right.$ and $\left.d_{4}\right)$ have a rotation angle of $30^{\circ}$ with respect to $d_{1}$ and $d_{2}$, respectively (see Figure 1). For the reference purpose, the results of perfect graphene are displayed as dashed lines in Figures 2. The S-W effect can either increase or 
decrease strain energy along different directions, and thus, it introduces the obvious anisotropy to graphene. For the structure with defect, $u_{1}>u_{3}>u_{4}>u_{g}>u_{2}$, where the subscripts $g$ and 1-4 represent perfect graphene and the structure with defect along four different directions $\left(d_{1}-d_{4}\right) . u_{1}$ is about 1.5 times of $u_{2}$, which means that graphene with a S-W defect is much stiffer along $d_{1}$ than $d_{2}$ direction.

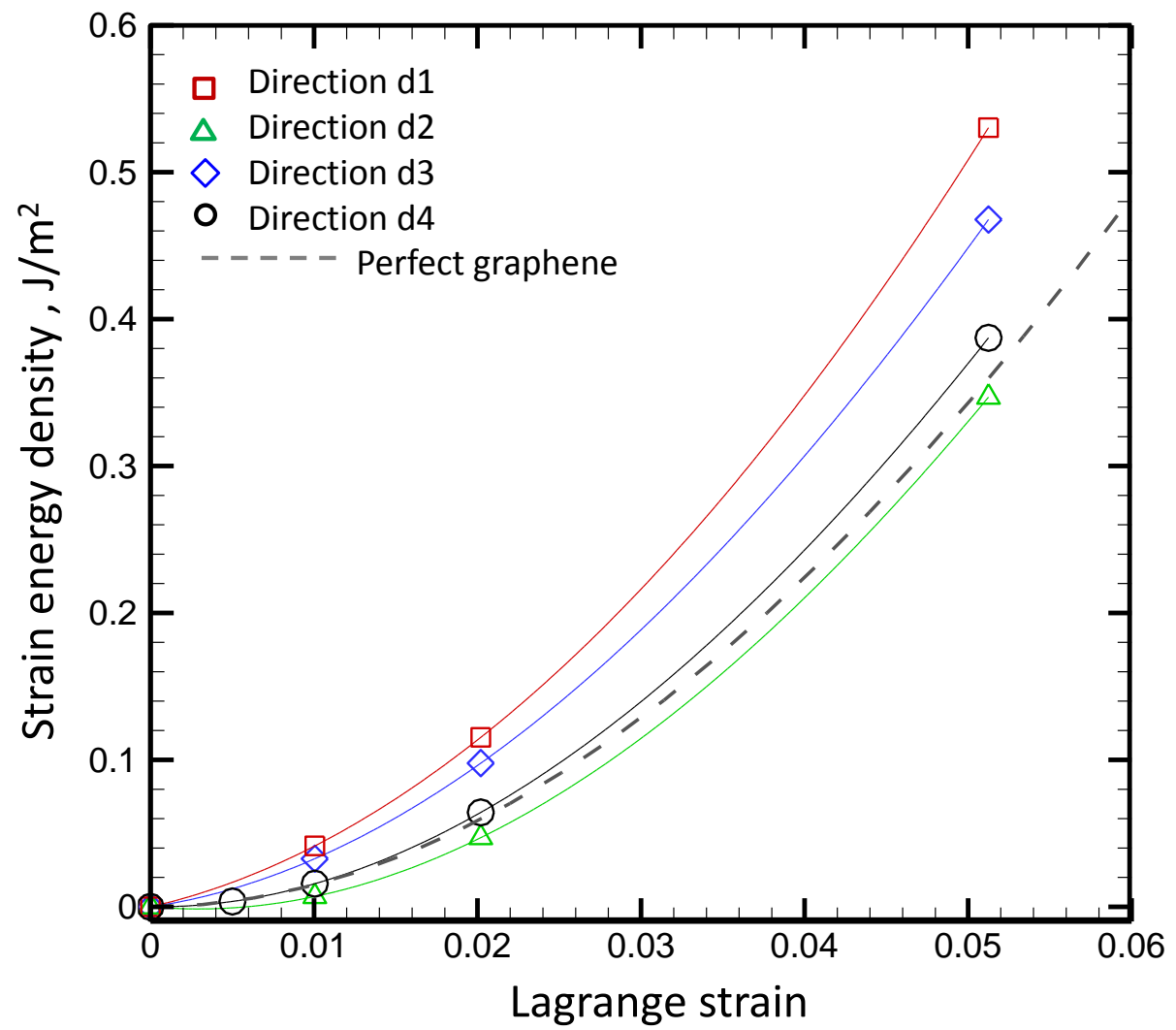

Figure 2. The relationship between the strain energy density and the Lagrange strain of graphene with a S-W defect along the different loading directions. The solid lines are fitting curves based on the equation of $u=\alpha \varepsilon^{2}+\beta \varepsilon^{3}+\sigma_{0} \varepsilon$; the dashed line is the result of perfect graphene.

Under a small tensile strain $(\varepsilon \leq 0.05)$, the $u$ of perfect graphene can be well fitted as a quadratic function of $\varepsilon: u=\alpha \varepsilon^{2}+\beta \varepsilon^{3}$ where $\alpha=2 E /(1-v)$ and $\beta$ is related to the nonlinear 
elastic constant of graphene under uniaxial strain stretching ( $E$ and $v$ are the elastic modulus and Poisson's ratio, respectively). However, the $u$ of graphene with $\mathrm{S}-\mathrm{W}$ defect does not follow the aforementioned equation, which is well fitted as: $u=\alpha \varepsilon^{2}+\beta \varepsilon^{3}+\sigma_{0} \varepsilon$ (displayed as the solid lines in Figure 2), where $\sigma_{0}$ can be considered to be the equivalent pre-stress created by the defect on the basis of continuum theory. Along the different directions of the structure with defect, the fitting parameters $(\alpha, \beta)$ are very close to each other as well as those of the prefect graphene. Thus, the S-W defect will not affect the values of $E$ and $v$ of graphene as well as the nonlinear elastic constant, whereas it will create the different values of $\sigma_{0}$ along different directions $\left(d_{1}-d_{4}\right): \sigma_{0}=2.370,-0.976,1.535,-0.147 \mathrm{~N} / \mathrm{m}$. Thus, the anisotropy of graphene with the S-W defect is mainly from the different pre-stress stiffing effect created by the defect. From the different values of $\sigma_{0}$, two 7-rings will create a pre-tension but two 5-rings create a pre-compression. It should be noted that $\sigma_{0}$ is the equivalent pre-stress of the overall computational cell based on the continuum theory, and the local pre-stress (close to 7-5 rings) will be much higher than this value.

It should be noted that the effect of the isolated $\mathrm{S}-\mathrm{W}$ defect on the mechanical property of graphene is investigated in the present work; if there are more defects appearing, the interaction between the defects will also affect the overall property of graphene (similar as the case of other type of 7-5 defects[28]), which will be studied in our future work.

\subsection{Effect of the defect on the tensile strength and failure strain}

The $\sigma-\varepsilon$ relationships along different directions are displayed in Figure 3, where $\sigma$ is the second-order Piola-Kirchhoff (PK2) stress. For the purpose of reference, the $\sigma-\varepsilon$ relationships 
of perfect graphene are also shown in Figure 3 (the dashed/dot-dash lines are for $z z / a c$ graphene, respectively): the tensile strengths $\sigma_{i n t}=28.2,29.8 \mathrm{~N} / \mathrm{m}$ and the failure strains are $\varepsilon_{f-g} \approx 0.3,0.6$ for ac/zz graphene based on DFT calculations, respectively. The failure strains $\left(\varepsilon_{f i}, i=1-4\right)$ of graphene with the defect along different directions $\left(d_{1}-d_{4}\right)$ range from $0.13-0.19\left(\varepsilon_{f 4}<\varepsilon_{f 3}=\varepsilon_{f 1}<\varepsilon_{f 2}\right)$. The failure strain under biaxial tension is very close to that along $d_{4}\left(\varepsilon_{f-b i} \approx \varepsilon_{f 4}\right)$, and thus, $d_{4}$ is the weakest direction of graphene with S-W defect.

The tensile strength $\sigma_{f}$ (the stress value corresponding to $\varepsilon_{f i}$ ) along different directions ranges from 25.7 (along $d_{4}$ ) to $28.2 \mathrm{~N} / \mathrm{m}$ (along $d_{2}$ ). Therefore, the $\mathrm{S}-\mathrm{W}$ defect just slightly decreases the tensile strength of graphene but significantly reduces the failure strain (i.e., the defect makes graphene much more brittle); in addition, the difference between the failure strain along $a c$ and $z z$ directions is also significantly reduced, e.g., the difference from $100 \%$ (for the perfect graphene) changes to about $20 \%$ (with a S-W defect). The present results further proved that the tensile strength/failure strain of graphene is not only dependent on the defect density, but also closely related the loading direction as well as the defect arrangement. 


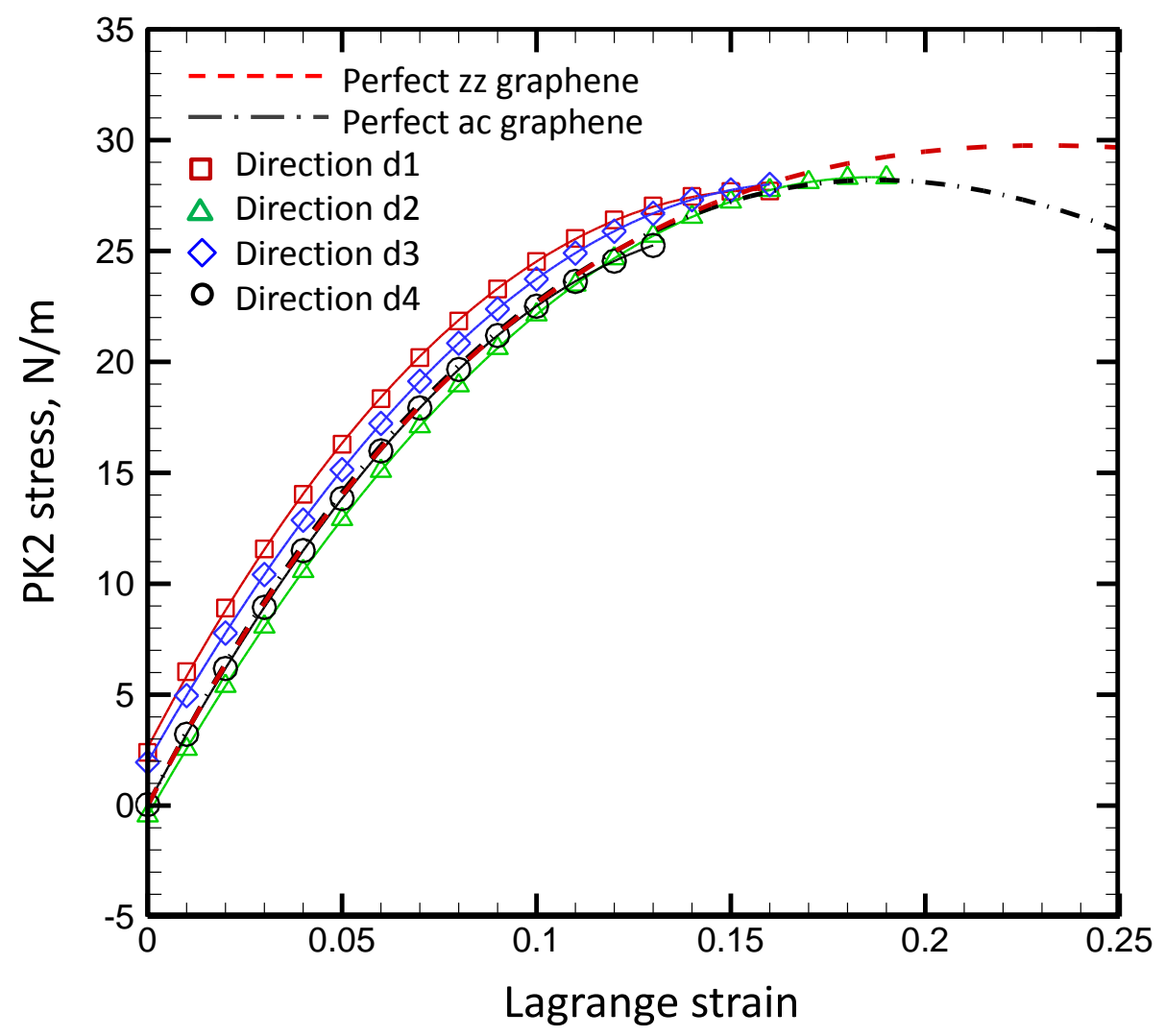

Figure 3. The relationship between the PK2 stress and the Lagrange strain of graphene with a S-W defect along the different loading directions. The dashed line and the dash-dot line are the results of the perfect graphene along $z z$ and $a c$ directions, respectively.

Based on the empirical AIREBO potential, He et al.[13] also reported that the tensile strength of graphene with the S-W defect is highly sensitive to the loading orientation: when the loading direction changes from $d_{2}(\mathrm{zz})$ to $d_{1}(\mathrm{ac})$, the value of $\sigma_{\text {int }}$ reduces from 36.1 to 4.0 $\mathrm{N} / \mathrm{m}$ and the corresponding failure strain $\varepsilon_{f}$ reduces from 0.17 to 0.02 ; when the loading direction changes from $d_{3}(\mathrm{zz})$ to $d_{4}(\mathrm{ac})$, the value of $\sigma_{\text {int }}$ reduces from 26.8 to $10.0 \mathrm{~N} / \mathrm{m}$ and the corresponding failure strain $\varepsilon_{f}$ reduces from 0.09 to 0.04 . Their results are highly different with the DFT results calculated in the present work. In addition, the decrease of $\sigma_{\text {int }}$ caused by the S-W defect should be lower than that caused by an isolated 7-5 defect $\left(\sigma_{\text {int }}=25.5 \mathrm{~N} / \mathrm{m}\right.$ 
from the MD simulations by Wei et al.[28]) since a pair of 7-5 defects with the opposite direction connected with each other (the S-W defect) should cause a lower pre-stretching of graphene due to the superposition effect of two 7-5 defects (The details of the pre-stress created by a S-W defect are shown in the next section). Thus, the empirical potential might be not accurate enough to calculate the failure behavior of graphene with the S-W defect.

By monitoring the Raman spectra of single walled carbon nanotube (SWCNT), Chang et al.[49] reported the experimental value of failure strain $\varepsilon_{f}=0.137$ which is much lower than its theoretical value calculated by the DFT but very close to the value calculated in the present work based on graphene with the S-W defect. Thus, the S-W defect might be the main reason to cause the big difference between the theoretical failure strain of graphene and its corresponding experimental value. Although we cannot prove that the S-W defect is the only defect owned by the SWCNT (it is typically to have different types of defects simultaneously), the S-W defect is easier to be created than other type of 7-5 defects due to its lower formation energy, which indicates that the probability of appearing S-W defect is higher than other type of 7-5 defects. In addition, our work is based on DFT calculations, and thus, it should be more accurate than other published numerical works based on the empirical potentials. Our work can be also used as the benchmark to check the effectiveness of the empirical simulations, and from this aspect, our work can provide an important help to understand the effect of other type defects on the failure stress of graphene.

\subsection{Pre-stress field of graphene induced by a $S$-W defect}


Currently, the well accepted mechanism of the effect of 7-5 defects on graphene failure is on the basis of the pre-tension induced by the defect and the C-C bond with the highest pre-tension is considered to be the weakest link which will be failed first. Thus, the structure with a higher pre-stretchered bond will have a lower tensile strength. For example, Wei et al.[28] shown that the strength of bi-crystal graphene is closely related to the maximum initial stress component along the loading direction; Grantab et al. [29] reported that the ultimate strength of graphene with 7-5 defects is controlled by the C-C bond with the highest initial strain (described by the bond stretching ratio) since it will be failed first.

The initial deformation of graphene introduced by a 7-5 defect can be estimated using the continuum theory based on the disclination dipoles model[28, 50]:

$$
\begin{aligned}
& \frac{\sigma_{x 0}}{\Gamma}=\frac{1}{2} \ln \frac{\left(x-x_{1}\right)^{2}+\left(y-y_{1}\right)^{2}}{\left(x-x_{2}\right)^{2}+\left(y-y_{2}\right)^{2}}+\frac{\left(y-y_{1}\right)^{2}}{\left(x-x_{1}\right)^{2}+\left(y-y_{1}\right)^{2}}-\frac{\left(y-y_{2}\right)^{2}}{\left(x-x_{2}\right)^{2}+\left(y-y_{2}\right)^{2}} \\
& \frac{\sigma_{y 0}}{\Gamma}=\frac{1}{2} \ln \frac{\left(x-x_{1}\right)^{2}+\left(y-y_{1}\right)^{2}}{\left(x-x_{2}\right)^{2}+\left(y-y_{2}\right)^{2}}+\frac{\left(x-x_{1}\right)^{2}}{\left(x-x_{1}\right)^{2}+\left(y-y_{1}\right)^{2}}-\frac{\left(x-x_{2}\right)^{2}}{\left(x-x_{2}\right)^{2}+\left(y-y_{2}\right)^{2}} \\
& \frac{\tau_{x y 0}}{\Gamma}=\frac{\left(x-x_{1}\right)\left(y-y_{1}\right)}{\left(x-x_{1}\right)^{2}+\left(y-y_{1}\right)^{2}}-\frac{\left(x-x_{2}\right)\left(y-y_{2}\right)}{\left(x-x_{2}\right)^{2}+\left(y-y_{2}\right)^{2}} \\
& \sigma_{01}=\frac{\sigma_{x 0}+\sigma_{y 0}}{2}+\sqrt{\left(\frac{\sigma_{x 0}-\sigma_{y 0}}{2}\right)^{2}+\tau_{x y 0}^{2}}
\end{aligned}
$$

where $\left(x_{1}, y_{1}\right)$ and $\left(x_{2}, y_{2}\right)$ are the location of the center of 5-ring and 7-ring, respectively; $\sigma_{x} 0$, $\sigma_{y 0}, \tau_{x y 0}$ are the components of the initial stress created by a 7-5 defect, and $\sigma_{01}$ is the maximum principle stress. $\Gamma=E \omega / 4 \pi, \omega$ is the rotational strength of the disclination (7-5 defect), and $\omega=21.8^{\circ}, 27.8^{\circ}$ for the armchair and zigzag directions, respectively. The stress field of the S-W defect can be considered as the simple superposition of a pair of 7-5 defects. With the value of $E$ of perfect graphene determined from DFT calculations ( $E=346.6,344.8$ 
$\mathrm{N} / \mathrm{m}$ along the $a c$ and $z z$ directions, respectively), the initial stress of each $\mathrm{C}-\mathrm{C}$ bond created by the defect can be calculated from its center coordinates $(x, y)$.

Figure 4 shows the calculated stress components created by a S-W defect along the loading directions $\sigma_{x 0}, \sigma_{y 0}$ and the shear component $\tau_{x y 0}$, as well as the maximum principal stress $\sigma_{10}$. Under the different loading directions $d_{1}, d_{2}, d_{3}$ and $d_{4}$, the bonds with the maximum initial stress component along the loading direction are following, respectively: h3 (or h4), h2 (or h5), h3 and h5 (the corresponding stress values are displayed in Table 1.
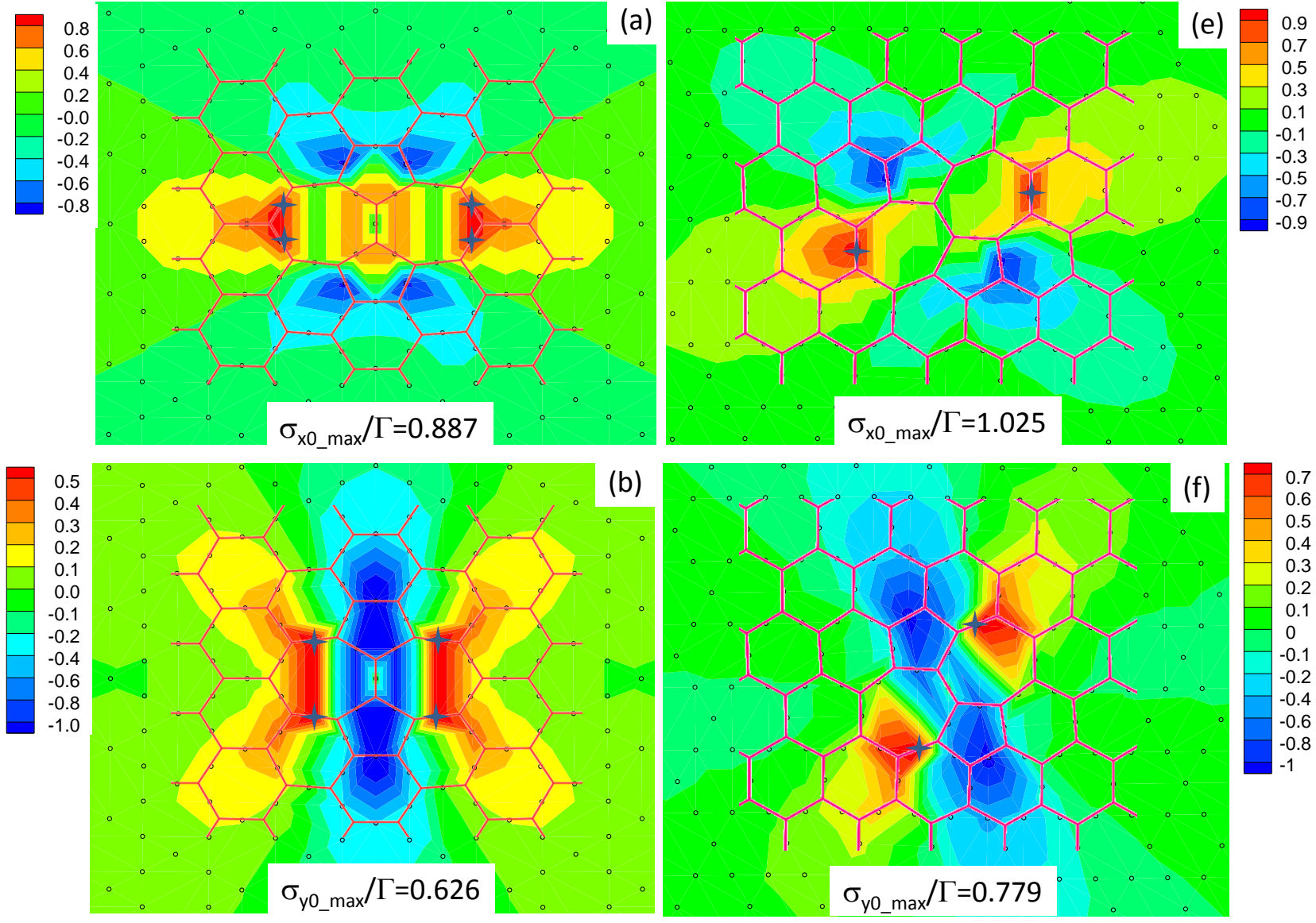

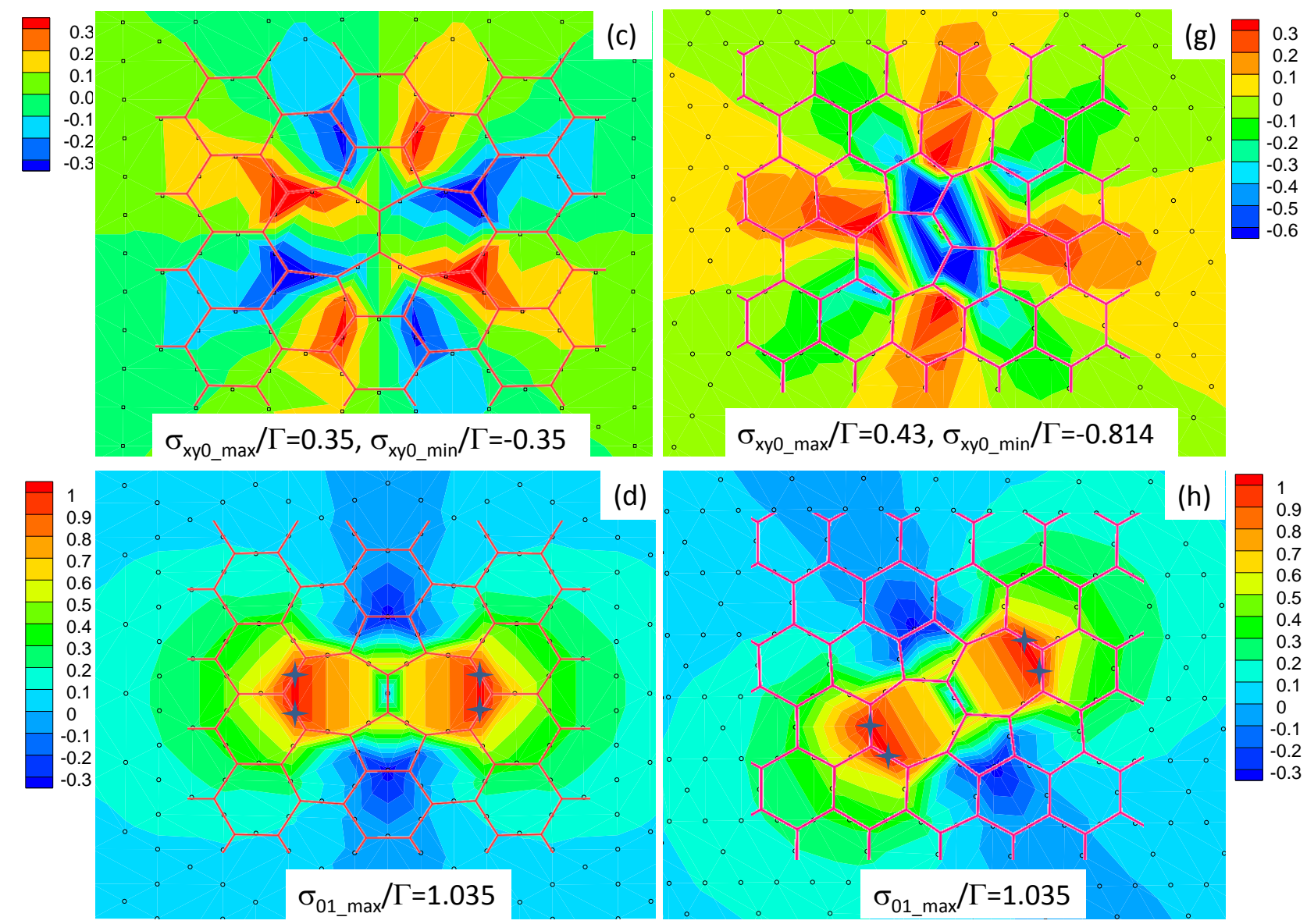

Figure 4. The normalized pre-stress components (created by a S-W defect) calculated by continuum theory on the basis of the declination model. (a)(e) $\sigma_{x 0} / \Gamma$; (b)(f) $\sigma_{y 0} / \Gamma ;$ (c) (g) $\tau_{x y 0} / \Gamma$; (d)(h) $\sigma_{01} / \Gamma$. (a)(b)(c)(d): $x$-, $y$-axis along $d_{1}, d_{2}$ direction; (e)(f)(g)(h): $x$-, $y$-axis along $d_{3}, d_{4}$ direction. The stars marked the bond with the maximum value.

The maximum initial stress component can be further calculated using MM simulations. In REBO potential, the pre-stress tensor components of each carbon atom (created by a S-W defect) can be calculated from the interaction force between this atom and its neighbors according to the following equation:

$$
\sigma_{a b}=-\frac{N}{A_{0} t}\left(\frac{1}{2} \sum_{j=1}^{n}\left(r_{i a} f_{i b}+r_{j a} f_{j b}\right)\right) \quad a, b=x, y
$$


where the subscripts $a, b$ represent the component along $x$ and $y$ directions (there are three components: $\sigma_{x x}, \sigma_{y y}$ and $\sigma_{x y}\left(\tau_{x y}\right)$ since graphene is $2 \mathrm{D}$ material, e.g., $\sigma_{a b}=\sigma_{x x}$ when $a=x, b=x$ represents the in-plane stress along $x$-direction), $n$ is the number of neighbor atoms of atom $i$ ( $n$ $=3$ for graphene), $A_{0}$ is the original computational cell area, $N$ is total number of carbon atoms in the computational cell and $t$ is the monolayer thickness $(t=0.335 \mathrm{~nm}), i, j$ are the atomic index of two atoms with the interaction, $r_{i a}, r_{i b}$ and $f_{i a}, f_{i b}$ are the coordinates and the interaction force vector components of atoms $i$ and $j$, respectively. Since the failure of graphene is actually controlled by the maximum C-C bond deformation (e.g., the critical breaking bond length reported by Wei et al.[28], $L_{c}=0.192 \mathrm{~nm}$ ), we focus on the C-C bond strength, which is approximately considered to be the average value of the stresses of two atoms associated with the bond.

Table 1 . The location and value of the maximum pre-stretching created by a S-W defect

\begin{tabular}{|c|c|c|c|c|}
\hline \multirow{2}{*}{ Method } & \multicolumn{4}{|c|}{ Loading direction } \\
\hline & $d_{1}$ & $d_{2}$ & $d_{3}$ & $d_{4}$ \\
\hline $\begin{array}{c}\text { Declination } \\
\text { model }\end{array}$ & h3(h4), $9.30 \mathrm{~N} / \mathrm{m}$ & h2(h5), $8.38 \mathrm{~N} / \mathrm{m}$ & $\mathrm{h} 3,13.71 \mathrm{~N} / \mathrm{m}$ & $\mathrm{h} 5,8.17 \mathrm{~N} / \mathrm{m}$ \\
\hline $\mathrm{MM}^{1}$ & h1(h6), $10.34 \mathrm{~N} / \mathrm{m}$ & h3(h4), 10.41N/m & $\mathrm{h} 3,10.57 \mathrm{~N} / \mathrm{m}$ & $\mathrm{h} 3,10.70 \mathrm{~N} / \mathrm{m}$ \\
\hline $\mathrm{MM}^{2}$ & h1(h6), $10.27 \mathrm{~N} / \mathrm{m}$ & h3(h4), $10.35 \mathrm{~N} / \mathrm{m}$ & $\mathrm{h} 3,10.49 \mathrm{~N} / \mathrm{m}$ & h3, $10.64 \mathrm{~N} / \mathrm{m}$ \\
\hline DFT* $^{*}$ & $\begin{array}{c}\text { h1(h6), } 0.0258 \\
(9.13 \mathrm{~N} / \mathrm{m})\end{array}$ & $\begin{array}{c}\mathrm{g} 1(\mathrm{~g} 3), 0.0221 \\
(7.97 \mathrm{~N} / \mathrm{m})\end{array}$ & $\begin{array}{c}\text { h1, } 0.0305 \\
(10.54 \mathrm{~N} / \mathrm{m})\end{array}$ & $\begin{array}{l}\mathrm{g} 3,0.0255 \\
(9.04 \mathrm{~N} / \mathrm{m})\end{array}$ \\
\hline
\end{tabular}

$\mathrm{MM}^{1}$ : from the large computational cell; $\mathrm{MM}^{2}$ : from the small computational cell.

*: The pre-strain values can be directly calculated by DFT and the corresponding pre-stress is determined from Eq. (4). 
Figures 5 shows the contour plots of the $\mathrm{C}-\mathrm{C}$ bond pre-stress components of graphene with a S-W defect calculated by MM simulations. The shapes of contour plots of the pre-stress components calculated from MM simulations are quite close to their theoretical counterpart (Figure 4), but the maximum values as well as their locations are different (as shown in Table 1). Except the tensile load along $d_{3}$ direction, the bonds with the largest pre-stress component calculated from MM simulations are different than their theoretical counterparts. From MM simulations, the maximum stress components of $\mathrm{C}-\mathrm{C}$ bonds under the different loading direction are very close to each other, whereas they are quite different from the theoretical calculation (the deviation is about $40 \%$ ).
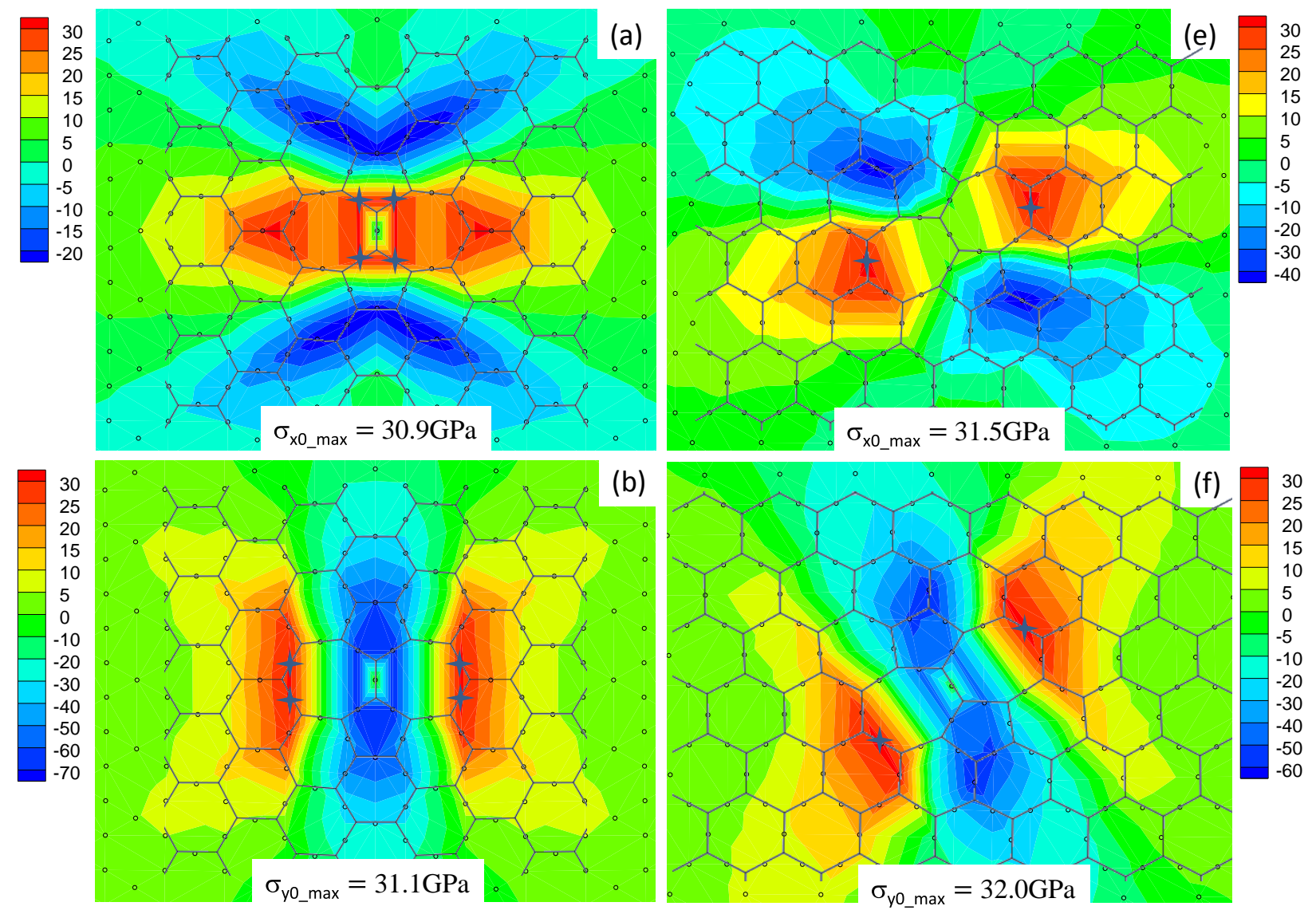

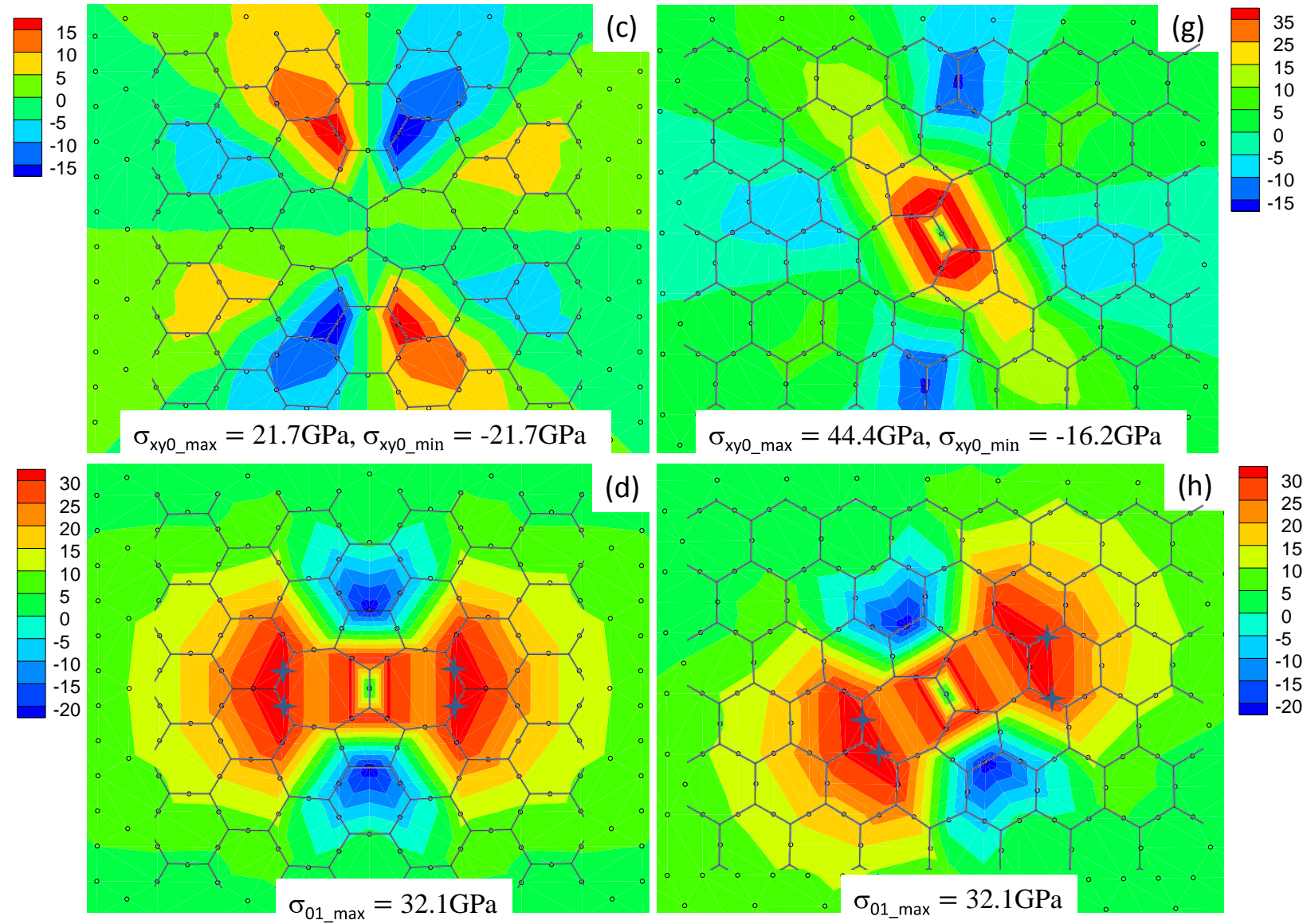

Figure 5. The pre-stress components (created by a S-W defect) calculated by MM simulations.

(a)(e) $\sigma_{x} 0 ;(\mathrm{b})(\mathrm{f}) \sigma_{y 0} ;(\mathrm{c})(\mathrm{g}) \sigma_{x y 0} ;(\mathrm{d})(\mathrm{h}) \sigma_{10} .(\mathrm{a})(\mathrm{b})(\mathrm{c})(\mathrm{d}): x$-, $y$-axis along $d_{l}, d_{2}$ direction;

$(\mathrm{e})(\mathrm{f})(\mathrm{g})(\mathrm{h}): x$-, $y$-axis along $d_{3}, d_{4}$ direction. The stars marked the bond with the maximum value.

Although DFT calculations cannot give the pre-stress values created by a S-W defect, they can provide the initial deformation on the basis of the pre-strain, which can be described by the bond length $(l)$ varying with respect to the initial bond length of perfect graphene $\left(l_{0}\right)$. In order to consider the influence of the loading direction, the pre-strain along the loading direction is defined as: 


$$
\begin{aligned}
& \varepsilon_{x 0}=\lambda_{0} \cos \theta, \varepsilon_{y 0}=\lambda_{0} \sin \theta \\
& \lambda_{0}=\frac{l-l_{0}}{l}
\end{aligned}
$$

where $\lambda_{0}$ is the initial bond stretching ratio, $l_{0}=0.1425 \mathrm{~nm}$ and $\theta$ is the angle between the bond and the loading direction. Figure 6 shows the contour plot of the initial deformation field $\left(\lambda_{0}, \varepsilon_{x 0}\right.$ and $\left.\varepsilon_{y 0}\right)$ created by a S-W defect. The shape of the contour plots of $\varepsilon_{x 0}$ and $\varepsilon_{y 0}$ are quite close to those of $\sigma_{x 0}, \sigma_{y 0}$ calculated by MM simulations (or the disclination dipoles model), but the locations of the maximum values of $\varepsilon_{x 0}$ and $\varepsilon_{y 0}$ are different with those of $\sigma_{x 0}, \sigma_{y 0}$ calculated from MM simulations or the theoretical solution, as shown in Table 1. Actually, the bond stiffness can be approximately estimated from the strain energy density $\left(u_{b i}\right)$ of perfect graphene under biaxial tension, in which all $\mathrm{C}-\mathrm{C}$ bonds have the same deformation. The bond stiffness is fitted as a quadratic function of the bond stretching ratio $\lambda[41]$ :

$$
k_{b o n d}=\frac{1}{n} \frac{\partial^{2} u_{b i}}{\partial \lambda^{2}} \approx A \lambda^{2}+B \lambda+C
$$

where $n$ is the number of bonds included in the computational cell, and $A, B$ and $C$ are fitting parameters $(A>0, B<0$ and $C>0)$. Based on Equation (4) and the initial bond stretching ratio (describe by Equation (3)), the pre-stress created by a S-W defect can be predicted by DFT calculations (displayed in Table 1), which are slightly lower than those obtained from MM simulations (10-20\% lower).

If the failure of graphene with a S-W defect is closely related to the bond with the maximum pre-deformation along the loading direction, the aforementioned difference in the pre-stress of bonds might be the reason for the different failure results predicted by the MM simulations and DFT calculations. Actually, although the overall load-deformation 
relationship obtained by the simulations based on the empirical potentials can be quite close to that determined from the DFT calculations, the stretching ratios of C-C bonds as well as the bond angle variation ratio are quite different from two methods[42]. The C-C-C bond angle strength is underestimated by the empirical potential in simulating the graphene deformation under uniaxial stretching (i.e., the bond angle can have a much larger change in MM simulations than that in DFT calculations), and consequently, the bond stretching ratio is also different from different methods. This difference might be caused by the electronic interactions which can be explicitly considered in DFT calculations but only implicitly shown by the fitted parameters in the empirical potentials. The decrease in C-C-C bond angle (created by two near sp2 bonds) will cause a rapid increase of the electronic repulsion which can be accurately described in DFT calculations but not in the empirical potentials, and thus, the bond angle variation strength is much lower in the MM simulations [42].

Since the value of $k_{b o n d}$ nonlinearly decreases with the increase of $\lambda$ (Equation (4)), the deformation of $\mathrm{C}-\mathrm{C}$ bond of graphene is highly nonlinear. Thus, a pre-tension of bond cannot only downshift the failure stress of bond by $\sigma_{0}$ but also couples with the non-linear elastic behavior to soften the bond (i.e., have a larger extension under the same load). The failure critical C-C bond length can be determined from biaxial tension $\left(L_{c}=0.19 \mathrm{~nm}\right.$ corresponding the critical bond stretching ratio $\lambda_{c}=0.33$, which is also very close to the critical breaking bond length reported by Wei et al. ( $\left.L_{c}=0.192 \mathrm{~nm}[28]\right)$. Therefore, the different bond pre-stress as well as the geometrical response to the uniaxial tensile deformation will lead to the different failure results determined from the different methods. In addition, 
The difference between the DFT results and the MM simulation results is not from the different computational cell size. We also calculate the pre-stress field from a small computational cell (including the same number of $\mathrm{C}$ atoms as that in DFT calculations), which is very similar to the results determined from the larger computational cell (displayed in Table 1). Thus, this might indicate that MM simulations (or the disclination dipoles model) will not accurately determine the intrinsic strength or the failure strain of graphene with the S-W defect. In addition, the results from the disclination dipoles model are also different from those calculated from MM simulations, which is different from the results of the aligned 7-5 defect reported by Wei et al.[28] This might indicate that the disclination dipoles model can only accurately describe the pre-stress field created by the very simple defect (e.g., the aligned 7-5 defects). 

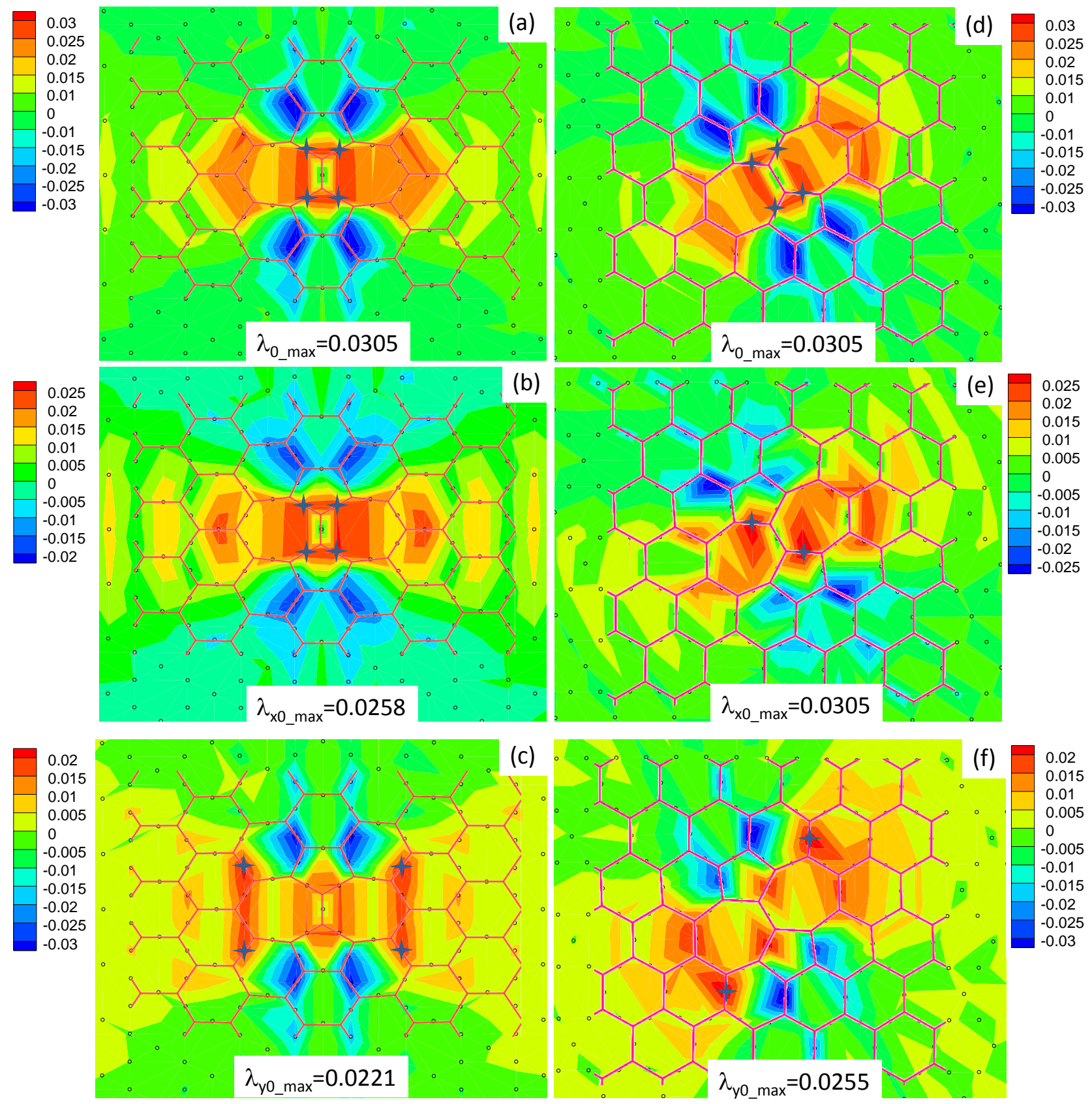

Figure 6. The pre-strain components (created by a S-W defect) calculated by DFT calculations.

(a)(d) $\lambda_{0} ;(\mathrm{b})(\mathrm{e}) \varepsilon_{x 0} ;$ (c)(f) $\varepsilon_{y 0}$; The stars marked the bond with the maximum value.

\section{Conclusions}

Using DFT calculations, we investigate the effect of the S-W defect on the mechanical properties of graphene under uniaxial/biaxial tension, and the pre-stress field created by the 
defect is also investigated. It is found that although the $\mathrm{S}-\mathrm{W}$ defect will significantly increase the anisotropy of graphene, it will not affect the elastic modulus and the Poisson's ratio as well as the nonlinear elastic constant of graphene. This anisotropy is mainly caused by the different pre-stress stiffing along the different direction, i.e., the S-W defect will create the different pre-stress along the different direction. In addition, the defect will significantly decrease the failure strain but slightly reduce the intrinsic strength.

Compared with the results determined from the MM simulations (or the continuum theory based on the disclination dipoles model), DFT calculations give the similar shape of the initial deformation field created by the S-W defect, but the location as well as the value of the maximum pre-stress are different. Due to the coupling effects of the different pre-stretching and tensile deformation response as well as the highly nonlinear bond stretching behavior, DFT calculations give the different intrinsic strength and the failure strain of graphene with the S-W defect. This also indicates that the MM simulations (or the continuum theory) will not accurately describe the failure behavior of graphene with the S-W defect. The present work can provide a useful guideline to understand the effects of defect on the tensile properties of graphene.

\section{Acknowledgments}

I acknowledge the financial support provided by the Ministry of Science and Technology of China (grant no. 2013CB933702) and the National Science Foundation of China (grant no. 11172002). 


\section{References:}

1. Geim, A.K., Graphene: Status and Prospects. Science, 2009. 324(5934): p. 1530-1534.

2. Novoselov, K.S., et al., Two-dimensional gas of massless Dirac fermions in graphene. Nature, 2005. 438(7065): p. 197-200.

3. Ferrari, A.C., et al., Raman spectrum of graphene and graphene layers. Physical Review Letters, 2006. 97(18): p. 187401.

4. Stankovich, S., et al., Graphene-based composite materials. Nature, 2006. 442(7100): p. 282-286.

5. Eda, G., G. Fanchini, and M. Chhowalla, Large-area ultrathin films of reduced graphene oxide as a transparent and flexible electronic material. Nature Nanotechnology, 2008. 3(5): p. 270-274.

6. Lee, C., X.D. Wei, J.W. Kysar, and J. Hone, Measurement of the elastic properties and intrinsic strength of monolayer graphene. Science, 2008. 321(5887): p. 385-388.

7. Soldano, C., A. Mahmood, and E. Dujardin, Production, properties and potential of graphene. Carbon, 2010. 48(8): p. 2127-2150.

8. Lee, G.D., et al., D(i)ffusion, coalescence, and reconstruction of vacancy defects in graphene layers. Physical Review Letters, 2005. 95(20): p. 205501.

9. Gass, M.H., et al., Free-standing graphene at atomic resolution. Nature Nanotechnology, 2008. 3(11): p. 676-681.

10. Zhu, J., M. He, and F. Qiu, Effect of Vacancy Defects on the Young's Modulus and Fracture Strength of Graphene: A Molecular Dynamics Study. Chinese Journal of Chemistry, 2012. 30(7): p. 1399-1404.

11. Meyer, J.C., et al., Direct Imaging of Lattice Atoms and Topological Defects in Graphene Membranes. Nano Letters, 2008. 8(11): p. 3582-3586.

12. Yazyev, O.V. and S.G. Louie, Topological defects in graphene: Dislocations and grain boundaries. Physical Review B, 2010. 81(19): p. 195420.

13. He, L.C., et al., The effect of Stone-Thrower-Wales defects on mechanical properties of graphene sheets - A molecular dynamics study. Carbon, 2014. 75: p. 124-132.

14. Grujicic, M., G. Cao, and R. Singh, The effect of topological defects and oxygen adsorption on the electronic transport properties of single-walled carbon-nanotubes. Applied Surface Science, 2003. 211(1-4): p. 166-183.

15. Warner, J.H., et al., Dislocation-Driven Deformations in Graphene. Science, 2012. 337(6091): p. 209-212.

16. Kotakoski, J., A.V. Krasheninnikov, U. Kaiser, and J.C. Meyer, From Point Defects in Graphene to Two-Dimensional Amorphous Carbon. Physical Review Letters, 2011. 106(10): p. 105505.

17. Grujicic, M., et al., UV-light enhanced oxidation of carbon nanotubes. Applied Surface Science, 2003. 214(1-4): p. 289-303.

18. Lahiri, J., et al., An extended defect in graphene as a metallic wire. Nature Nanotechnology, 2010. 5(5): p. 326-329. 
19. Lu, J., Y. Bao, C.L. Su, and K.P. Loh, Properties of Strained Structures and Topological Defects in Graphene. Acs Nano, 2013. 7(10): p. 8350-8357.

20. Banhart, F., J. Kotakoski, and A.V. Krasheninnikov, Structural Defects in Graphene. Acs Nano, 2011. 5(1): p. 26-41.

21. Lee, G.H., et al., High-Strength Chemical-Vapor Deposited Graphene and Grain Boundaries. Science, 2013. 340(6136): p. 1073-1076.

22. Kim, P., GRAPHENE Across the border. Nature Materials, 2010. 9(10): p. 792-793.

23. Yu, Q.K., et al., Control and characterization of individual grains and grain boundaries in graphene grown by chemical vapour deposition. Nature Materials, 2011. 10(6): p. 443-449.

24. Gibb, A.L., et al., Atomic Resolution Imaging of Grain Boundary Defects in Monolayer Chemical Vapor Deposition-Grown Hexagonal Boron Nitride. Journal of the American Chemical Society, 2013. 135(18): p. 6758-6761.

25. Huang, P.Y., et al., Grains and grain boundaries in single-layer graphene atomic patchwork quilts. Nature, 2011. 469(7330): p. 389-+.

26. Ruiz-Vargas, C.S., et al., Softened Elastic Response and Unzipping in Chemical Vapor Deposition Graphene Membranes. Nano Letters, 2011. 11(6): p. 2259-2263.

27. Rasool, H.I., et al., Measurement of the intrinsic strength of crystalline and polycrystalline graphene. Nature Communications, 2013. 4: p. 2811.

28. Wei, Y.J., et al., The nature of strength enhancement and weakening by pentagon-heptagon defects in graphene. Nature Materials, 2012. 11(9): p. 759-763.

29. Grantab, R., V.B. Shenoy, and R.S. Ruoff, Anomalous Strength Characteristics of Tilt Grain Boundaries in Graphene. Science, 2010. 330(6006): p. 946-948.

30. Zhang, T., X.Y. Li, and H.J. Gao, Defects controlled wrinkling and topological design in graphene. Journal of the Mechanics and Physics of Solids, 2014. 67: p. 2-13.

31. Mortazavi, B. and G. Cuniberti, Atomistic modeling of mechanical properties of polycrystalline graphene. Nanotechnology, 2014. 25(21): p. 215704.

32. Liu, Y.Y. and B.I. Yakobson, Cones, Pringles, and Grain Boundary Landscapes in Graphene Topology. Nano Letters, 2010. 10(6): p. 2178-2183.

33. Song, Z.G., V.I. Artyukhov, B.I. Yakobson, and Z.P. Xu, Pseudo Hall-Petch Strength Reduction in Polycrystalline Graphene. Nano Letters, 2013. 13(4): p. 1829-1833.

34. Sha, Z.D., et al., Inverse Pseudo Hall-Petch Relation in Polycrystalline Graphene. Scientific Reports, 2014. 4: p. 5991.

35. Zhang, J.F., J.J. Zhao, and J.P. Lu, Intrinsic Strength and Failure Behaviors of Graphene Grain Boundaries. Acs Nano, 2012. 6(3): p. 2704-2711.

36. Han, J., S. Ryu, D. Sohn, and S. Im, Mechanical strength characteristics of asymmetric tilt grain boundaries in graphene. Carbon, 2014. 68: p. 250-257.

37. Jhon, Y.I., S.E. Zhu, J.H. Ahn, and M.S. Jhon, The mechanical responses of tilted and non-tilted grain boundaries in graphene. Carbon, 2012. 50(10): p. 3708-3716.

38. Wu, J.T. and Y.J. Wei, Grain misorientation and grain-boundary rotation dependent mechanical properties in polycrystalline graphene. Journal of the Mechanics and Physics of Solids, 2013. 61(6): p. 1421-1432. 
39. Kotakoski, J. and J.C. Meyer, Mechanical properties of polycrystalline graphene based on a realistic atomistic model. Physical Review B, 2012. 85(19): p. 195447.

40. Zhou, L.X., Y.G. Wang, and G.X. Cao, Elastic properties of monolayer graphene with different chiralities. Journal of Physics-Condensed Matter, 2013. 25(12): p. 125302.

41. Cao, G.X., Atomistic Studies of Mechanical Properties of Graphene. Polymers, 2014. 6(9): p. 2404-2432.

42. Zhou, L. and G. Cao, Nonlinear anisotropic deformation behavior of graphene monolayer under uniaxial tension Physical Chemistry Chemical Physics 2015. 18: p. 1657.

43. Kresse, G. and J. Furthmuller, Efficient iterative schemes for ab initio total-energy calculations using a plane-wave basis set. Physical Review B, 1996. 54(16): p. 11169-11186.

44. Kresse, G. and J. Furthmuller, Efficiency of ab-initio total energy calculations for metals and semiconductors using a plane-wave basis set. Computational Materials Science, 1996. 6(1): p. 15-50.

45. Perdew, J.P., K. Burke, and M. Ernzerhof, Generalized gradient approximation made simple. Physical Review Letters, 1996. 77(18): p. 3865-3868.

46. Saito, R., M. Fujita, G. Dresselhaus, and M.S. Dresselhaus, Physical Properties of Carbon Nanotubes. 1998, London: Imperial College London:.

47. Wei, Y.J., et al., Bending Rigidity and Gaussian Bending Stiffness of Single-Layered Graphene. Nano Letters, 2013. 13(1): p. 26-30.

48. Plimpton, S., Fast Parallel Algorithms for Short-Range Molecular-Dynamics. Journal of Computational Physics, 1995. 117(1): p. 1-19.

49. Chang, C.C., et al., A New Lower Limit for the Ultimate Breaking Strain of Carbon Nanotubes. Acs Nano, 2010. 4(9): p. 5095-5100.

50. Li, J.C.M., Disclination Model of High Angle Grain-Boundaries. Surface Science, 1972. 31(1): p. 12-\&. 